\title{
A Numerical Study on Electrical Percolation of Polymer-Matrix Composites with Hybrid Fillers of Carbon Nanotubes and Carbon Black
}

\author{
Yuli Chen, Shengtao Wang, Fei Pan, and Jianyu Zhang \\ Institute of Solid Mechanics, Beihang University, Beijing 100191, China \\ Correspondence should be addressed to Jianyu Zhang; jyzhang@buaa.edu.cn
}

Received 28 July 2013; Accepted 22 January 2014; Published 9 March 2014

Academic Editor: Fathallah Karimzadeh

Copyright (C) 2014 Yuli Chen et al. This is an open access article distributed under the Creative Commons Attribution License, which permits unrestricted use, distribution, and reproduction in any medium, provided the original work is properly cited.

\begin{abstract}
The electrical percolation of polymer-matrix composites (PMCs) containing hybrid fillers of carbon nanotubes (CNTs) and carbon black (CB) is estimated by studying the connection possibility of the fillers using Monte Carlo simulation. The 3D simulation model of CB-CNT hybrid filler is established, in which CNTs are modeled by slender capped cylinders and CB groups are modeled by hypothetical spheres with interspaces because $\mathrm{CB}$ particles are always agglomerated. The observation on the effects of CB and CNT volume fractions and dimensions on the electrical percolation threshold of hybrid filled composites is then carried out. It is found that the composite electrical percolation threshold can be reduced by increasing CNT aspect ratio, as well as increasing the diameter ratio of CB groups to CNTs. And adding CB into CNT composites can decrease the CNT volume needed to convert the composite conductivity, especially when the CNT volume fraction is close to the threshold of PMCs with only CNT filler. Different from previous linear assumption, the nonlinear relation between $\mathrm{CB}$ and $\mathrm{CNT}$ volume fractions at composite percolation threshold is revealed, which is consistent with the synergistic effect observed in experiments. Based on the nonlinear relation, the estimating equation for the electrical percolation threshold of the PMCs containing CB-CNT hybrid fillers is established.
\end{abstract}

\section{Introduction}

Polymers have been widely used in industrial manufactures, military equipment, and daily life due to their excellent plasticity, lightweight, and low cost. However, most polymers are electrically isolated, which strongly limits their applications in the fields requiring antistatic, electrostatic dissipative, and electromagnetic shielding performances. The inherently conductive polymers are usually expensive and easily aging [1], while adding electrical conductive fillers into polymers can also make them conductive and may improve their mechanical properties as well. Therefore, adding conductive fillers into polymers is an attractive way to enhance conductivity of the polymers [2].

Carbon nanotubes (CNTs) have been considered as an ideal reinforcement phase in composites due to their excellent mechanical and physical properties [3-6]. Especially, their high aspect ratio (over 1000) and high conductivity (up to $10^{4} \mathrm{~S} / \mathrm{cm}$ ) enable them to greatly improve the conductivity of polymer-matrix composites (PMCs) with a very low volume fraction [7-9]. A large number of experiments have been carried out to study the electrical percolation threshold of CNT/polymer composites [4-14]. Meanwhile, theoretical and numerical studies have also been done to estimate the percolation threshold of CNT/polymer composites [15-24], in which excluded volume method [15] and Monte Carlo simulation [17-21] are widely accepted. All these studies indicate that better dispersion, larger aspect ratio, and less waviness of CNTs lead to a lower percolation threshold. However, CNTs with large aspect ratio are easily agglomerated and entangled when dispersing in polymer matrixes [25-27], so it is difficult to get a percolation threshold as low as estimated in simulations. Moreover, the high price of CNTs also limits their application to some extent. Therefore, hybrid filling becomes a good solution to these problems. Carbon black (CB) is one of the most widely used conductive nanoparticles in industrial applications so far and thus considered as a suitable candidate because of its low cost, good dispersion 


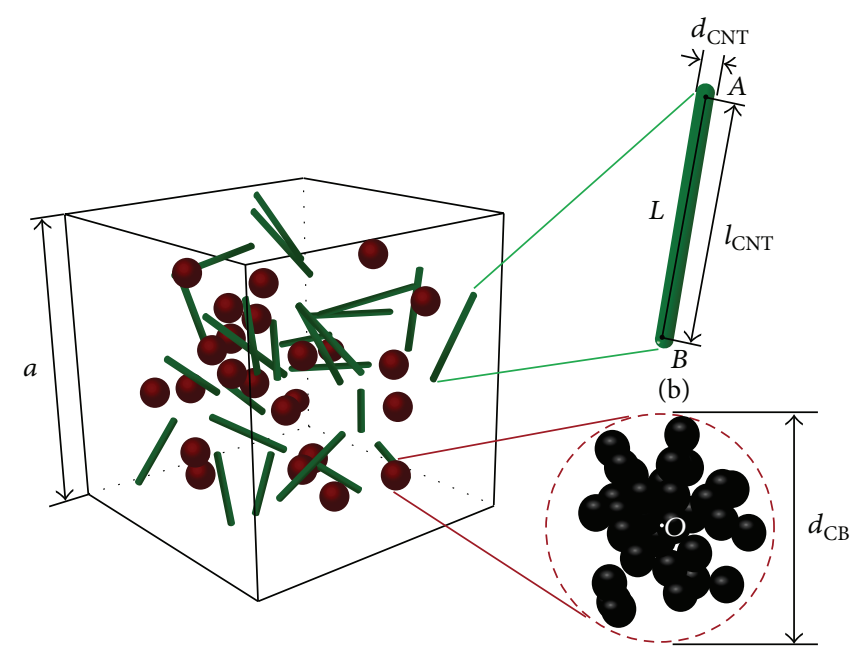

(a)

(c)

FIGURE 1: Simulation model: (a) representative volume element for CB-CNT/polymer composites, (b) spherocylinder model for CNT filler, and (c) hypothetical sphere model for agglomerated CB particles.

ability, and especially synergistic effect with CNTs which can further improve conductivity and other properties of PMCs. Experimental studies have shown the advantages of the synergistic effect of CB. For example, Socher et al. [1] observed the synergistic effects of $\mathrm{CB}$ in multiwalled CNTs (MWCNTs) and CB hybrid nanocomposites regarding maximum conductivity and MWCNT dispersion. Sumfleth et al. [28] found synergistic effects in network formation and in charge transport when they studied conductivity and microstructure of ternary $\mathrm{CB} / \mathrm{CNTs}$-epoxy system. Ma et al. [8] also studied CB/CNTs-epoxy composites and found that besides the synergistic effects on conductivity the hybrid fillers improved ductility and fracture toughness of composites more than equivalent $\mathrm{CB}$ or CNTs.

However, the experimental studies on the PMC with hybrid fillers are hardly capable of predicting the electrical percolation systematically or quantitatively. According to experiments, researchers can only guess the relation between amount of fillers and electrical percolation of composites and may draw some different, even opposite conclusions. Therefore, it is necessary to develop a theoretical simulation approach to guide the experimental trials. So far, existing numerical and theoretical methods for conductivity and percolation threshold of PMCs are almost all about pure CNT fillers and simulation models are established for the CNT fillers. For the composites with hybrid fillers, as proved in the experiment studies, the fillers can not only combine advantages of different fillers but also improve material properties by synergistic effects. So adding hybrid fillers into PMCs could be potential and promising to enhance the conductivity and other properties of composites.

This paper aims to better understand the synergistic effect and systematically study the effecting factors on electrical percolation threshold of CB-CNT hybrid filler, including $\mathrm{CB}$ and $\mathrm{CNT}$ volume fractions, aspect ratio of CNTs, and diameter ratio of $\mathrm{CB}$ groups to CNTs. A 3D simulation model for the CB-CNT hybrid filler is set up and a nonlinear law to estimate the percolation threshold is obtained, in which the synergistic effect between $\mathrm{CB}$ and CNT volume fractions as well as the effects of geometric dimensions of CB groups and CNTs is taken into account.

\section{Numerical Simulation}

For PMCs with conductive fillers, the electrical percolation threshold is dominated by the network formation and interactions of the electrical conductive fillers because the conductivity of the polymer matrix is much lower than that of the fillers and can be ignored in the simulation. If the electrical conductive fillers can form conducting pathways to transport electrons, the composite is electrical conductive. With increasing volume fraction of the fillers, the possibility to form these pathways increases. Once the conductive pathway forms, the composite conductivity increases significantly, and this filler volume fraction to form the conductive pathway is called electrical percolation threshold. Therefore, it can be assumed that the electrical percolation threshold is related to the possibility to form the conductive pathway by the fillers. This section will try to estimate the electrical percolation threshold of PMCs with CB-CNT hybrid fillers by calculating the possibility of the pathway formation, and Monte Carlo method is adopted in the numerical simulation.

2.1. 3D Simulation Models for CNTs and CB. For PMCs with CB-CNT hybrid fillers, the simulation models for both CNT and $\mathrm{CB}$ are needed in Monte Carlo simulation. In PMCs containing CNT only, the slender spherocylinder model illustrated in Figure 1(b) is commonly used to simulate the CNTs and hence is adopted in this study for the CNT phase. The diameter and length of cylinder model are equal to those of CNT filler, respectively. 
A single $\mathrm{CB}$ particle is a sphere with diameters from several to tens of nanometers. Agglomerated CB particles always construct intertwined chains, as shown in Figure 1(c). Suppose that the lengths of the intertwined chains are statistically the same in all directions, which means one group of $\mathrm{CB}$ particles can connect to any other group of $\mathrm{CB}$ particles within the scope of the chain length in all directions in the space, so from the view of pathway connection, a group of CB particles can be equivalent to a hypothetical sphere with the diameter equal to the average chain length, as presented by the dashed circle in Figure 1(c). It should be noted that the hypothetical sphere is not filled with $\mathrm{CB}$ only but with both $\mathrm{CB}$ and the polymer matrix. The volume fraction of the polymer matrix in the hypothetical sphere is assumed as $70 \%$ in this study. This hypothetical sphere model can also be used to simulate intertwined and agglomerated CNT fillers.

It is easy to involve both slender spherocylinder and hypothetical sphere models in Monte Carlo simulation, as shown in Figure 1(a), which presents a 3D cubic representative volume element (RVE) for the simulation of PMCs with randomly distributed CNTs and $\mathrm{CB}$ groups. More other shapes/models can be easily taken into account in the simulation, but in this paper, only the two models are used to simulate the two types of fillers, CNTs and CB, which are very promising to be applied in PMCs as mentioned above.

It should be noticed that CNTs are not penetrable to each other in actual composites, but they can superpose on each other in simulations. Berhan and Sastry $[18,19]$ figured out that the error could exceed $4 \%$ for CNTs with aspect ratio less than 400 if impenetrableness was not taken into consideration. In this study, the overlapped volume is deducted from the total volume of CNTs to eliminate the error of penetration between CNT models. But for the hypothetical sphere models of agglomerated CB particles, it is reasonable to think they are penetrable because when they are getting close the $\mathrm{CB}$ chains in the hypothetical sphere can adjust themselves to avoid penetrating due to the interspaces between the chains, which are taken into account in the volume of the hypothetical sphere model as matrix volume. And for the same reason, when a CNT model penetrates in a $\mathrm{CB}$ model, the overlapped volume need not be deducted. In another word, the impenetrableness is necessary to be taken into consideration only when the CNTs superpose on each other.

2.2. Numerical Implementation. To form the conductive pathway, the fillers need to make contact with each other first. Here the contact includes not only geometrical contact but also physical contact such as electron tunneling, which means two particles are close enough to let electrons skip although they do not geometrically contact with each other. Whether the pathway exists or not can be determined according to all the contacts between fillers. Repeating the calculation for times can obtain the possibility to form the pathway, which is defined as "connection possibility" in this study. The connection possibility can be used to evaluate the electrical percolation threshold. The essential steps to calculate the connection possibility of the PMCs containing both CNT and CB fillers are described below.

(1) Initialize variables: the connection times $N_{C}=0$ and the total simulation times $N_{T}=0$.

(2) For given volume fractions and dimensions of CNTs and $\mathrm{CB}$, generate slender spherocylinder and hypothetical sphere models, respectively, in a cubic RVE. The position and direction of the CNT model are totally random and represented by its axial line segment $L$ with two end points $A$ and $B$, as shown in Figure 1(b). The position of CB model is also random and represented by its center point $O$, as shown in Figure 1(c).

(3) According to the positions generated in step 1, evaluate the intersections between every two models by calculating the distances between them, and then build an intersection data matrix. The criterion to evaluate intersection between fillers $i$ and $j$ is

$$
D_{i j} \leq \frac{\left(d_{i}+d_{j}\right)}{2}+d_{\text {tunnel }} .
$$

Here $d$ is the diameter of the spherocylinder for CNTs or the hypothetical sphere for CB, $d_{\text {tunnel }}$ is the largest distance for tunnel effect and $d_{\text {tunnel }}=0$ if the tunnel effect is excluded, and $D_{i j}$ is the distance between fillers $i$ and $j$.

(4) Check the intersection data matrix to find a pathway from one side to the opposite side of the RVE. This pathway is considered as a route along which electrons can be transported. If the pathway can be found, update the connection times as $N_{C}=N_{C}$ +1 ; otherwise, $N_{C}$ remains unchanged. The total simulation times $N_{T}$ are also updated as $N_{T}=N_{T}+$ 1 no matter the RVE is connected or not.

(5) Repeat steps $2 \sim 4$ for times to determine the connection of the RVEs with the same filler volume fractions and filler dimensions but different random distributions, and then calculate the connection possibility $\mathrm{CP}$ as $\mathrm{CP}=N_{C} / N_{T}$.

2.3. Connection Possibility and Its Sensitivity to RVE Size. From the procedure in Section 2.2, the connection possibility for given filler volume fraction and filler dimensions is obtained. Changing the filler volume fraction, the relation between the connection possibility and the filler volume fraction can be depicted, as shown in Figure 2. Figure 2(a) shows the simulation result of CNT/polymer composites. The diameter and length of CNT fillers, $d_{\mathrm{CNT}}$ and $l_{\mathrm{CNT}}$, are $2 \mathrm{~nm}$ and $200 \mathrm{~nm}$, respectively, and the side length of the cubic RVE, $a$, is set to be $600 \mathrm{~nm}, 800 \mathrm{~nm}$, and $1000 \mathrm{~nm}$. Figure 2(b) presents the simulation result of $\mathrm{CB} /$ polymer composites. The diameter of CB groups, $d_{\mathrm{CB}}$, is $6 \mathrm{~nm}$, and the side length of the RVE, $a$, is chosen as $60 \mathrm{~nm}, 120 \mathrm{~nm}$, and $240 \mathrm{~nm}$. The distance for tunnel effect $d_{\text {tunnel }}=0$ because of the low filler volume fraction. 


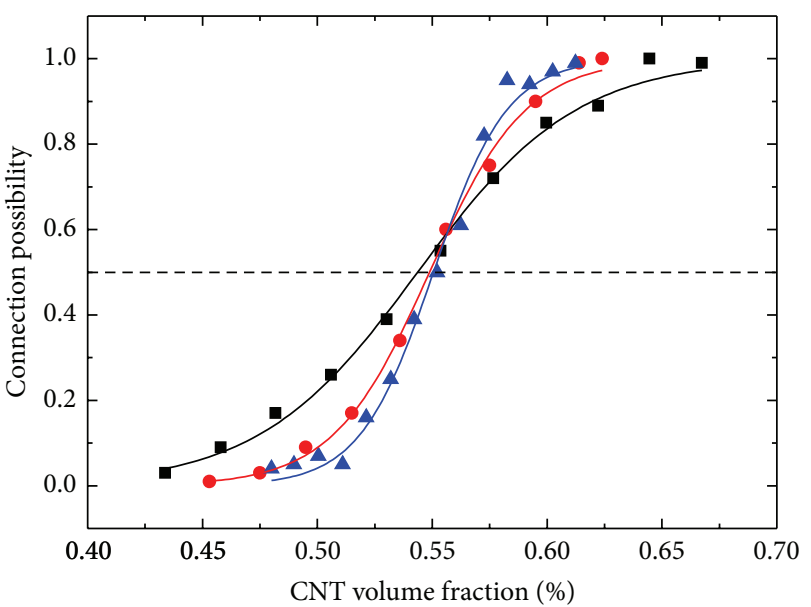

- $a / l_{\mathrm{CNT}}=3$

- $a / l_{\mathrm{CNT}}=4$

$\Delta a / l_{\mathrm{CNT}}=5$

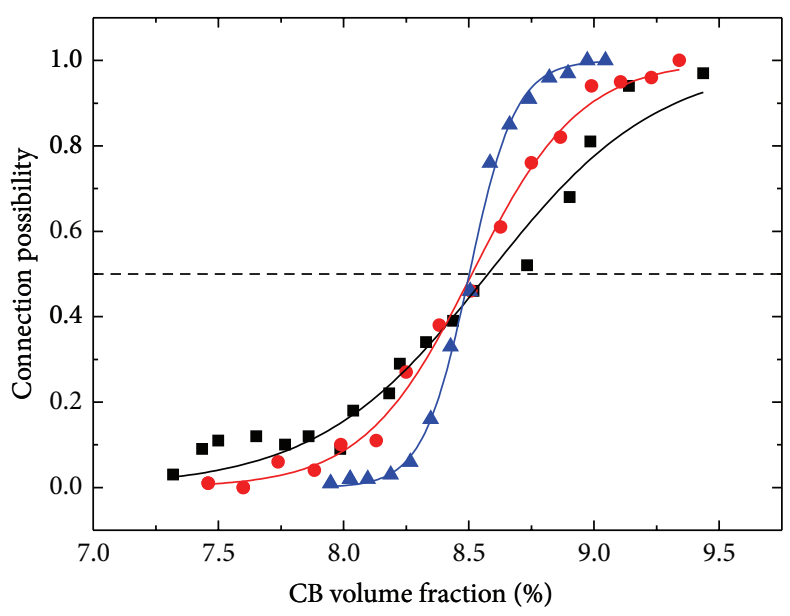

- $a / d_{\mathrm{CB}}=10$

- $a / d_{\mathrm{CB}}=20$

$\Delta a / d_{\mathrm{CB}}=40$

(a)

(b)

FIGURE 2: Effect of RVE size $a$ on connection possibility for (a) CNT/polymer composites $\left(d_{\mathrm{CNT}}=2 \mathrm{~nm}, l_{\mathrm{CNT}}=200 \mathrm{~nm}\right)$ and (b) CB/polymer composites $\left(d_{\mathrm{CB}}=6 \mathrm{~nm}\right)$. The solid curves are obtained from fitting of Boltzmann function.

TABLE 1: Parameters of Boltzmann fitting for connection possibilities.

\begin{tabular}{lccccc}
\hline & \multicolumn{3}{c}{ PMCs with CNT only } & \multicolumn{3}{c}{ PMCs with CB only } \\
$a / l_{\mathrm{CNT}}$ & $\Phi_{0}(\%)$ & $K$ & $a / d_{\mathrm{CB}}$ & $\Phi_{0}(\%)$ & $K$ \\
\hline 3 & 0.551 & 652.36 & 10 & 8.68 & 49.02 \\
4 & 0.553 & 1074.46 & 20 & 8.53 & 109.17 \\
5 & 0.548 & 1576.61 & 40 & 8.50 & 282.65 \\
\hline
\end{tabular}

TABLE 2: Comparison between $\Phi_{0}$ and percolation thresholds obtained from excluded volume method [15] for different CNT aspect ratios in $\mathrm{CNT} /$ polymer composites.

\begin{tabular}{lccc}
\hline Aspect ratio & $\Phi_{0}$ & Percolation thresholds & Relative error \\
\hline 300 & 0.178 & 0.165 & 0.0788 \\
500 & 0.105 & 0.0993 & 0.0574 \\
750 & 0.0687 & 0.0664 & 0.0346 \\
1000 & 0.0495 & 0.0498 & 0.0060 \\
\hline
\end{tabular}

Both figures show that the connection possibility first has little response to the filler volume fraction and then suddenly increases sharply with increasing filler volume fraction and saturates quickly. This behavior can be described by Boltzmann function as

$$
\mathrm{CP}(\Phi)=A_{2}-\frac{A_{2}-A_{1}}{1+\exp \left[4 K\left(\Phi-\Phi_{0}\right) /\left(A_{2}-A_{1}\right)\right]} .
$$

Here $A_{1}$ and $A_{2}$ are the minimum and maximum values of the function $\mathrm{CP}(\Phi)$, respectively, so for the function of connection possibility, $A_{1}=0$ and $A_{2}=1$. $\Phi$ represents the volume fraction of the filler, and $\Phi_{0}$ is the $\Phi$ value satisfying $\mathrm{CP}(\Phi)=\left(A_{2}-A_{1}\right) / 2 . K$ is the slope of function $\mathrm{CP}(\Phi)$ at $\Phi$ $=\Phi_{0}$. Thus, every curve in Figure 2 can be described by $\Phi_{0}$, the volume fraction for $50 \%$ connection possibility, and $K$, the slope of the curve at $\Phi_{0}$, which are listed in Table 1 .

Figure 2 also indicates that the simulation results of connection possibility are very sensitive to the size of the RVE. The slope of the curve $K$ depends strongly on the RVE size, becoming steeper with increasing RVE size, as shown in Table 1. So in order to get an accurate result by Monte Carlo simulation, the simulation region must be big enough to contain a large number of fillers.

However, in both Figures 2(a) and 2(b), the curves meet together when the connection possibility is about $50 \%$, which means $\Phi_{0}$ can be hardly affected by the size of the RVE, as presented quantitatively in Table 1 . Therefore, $\Phi_{0}$ is selected to represent the electrical percolation threshold, so that the RVE size is no longer an influence factor on the result. In this case, it is not necessary to choose a very large simulation region, which absolutely leads to more CPU time in simulations.

Taking into account both accuracy and efficiency, $\Phi_{0}$ is selected to represent the electrical percolation threshold, and $a / d_{\mathrm{CB}} \geq 30$ and $a / l_{\mathrm{CNT}} \geq 5$ are applied in the following simulations.

\subsection{Relation between Connection Possibility and Percolation} Threshold. To establish the relation between the electrical percolation threshold and $\Phi_{0}$, the filler volume fraction for $50 \%$ connection possibility, comparison between simulation results and theoretical values based on excluded volume method [15] for PMCs containing only CNTs is made. In the comparison, the diameter of CNT model is $2 \mathrm{~nm}$, and four different aspect ratios $\left(l_{\mathrm{CNT}} / d_{\mathrm{CNT}}\right) 300,500,750$, and 1000 are studied. Table 2 shows the comparison between $\Phi_{0}$ and the electrical percolation threshold estimated by excluded volume method [15]. It can be seen that $\Phi_{0}$ is very close 


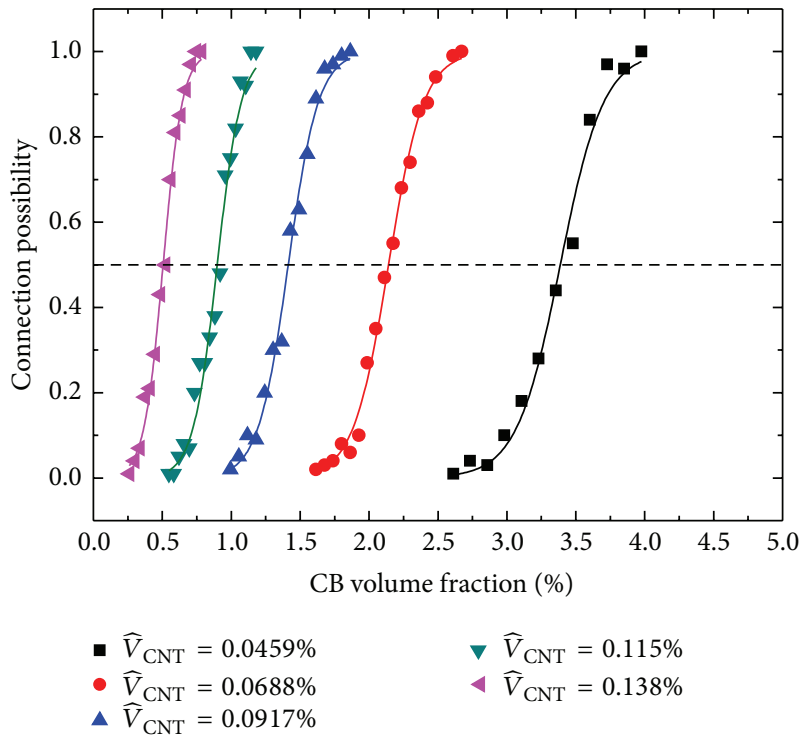

(a)

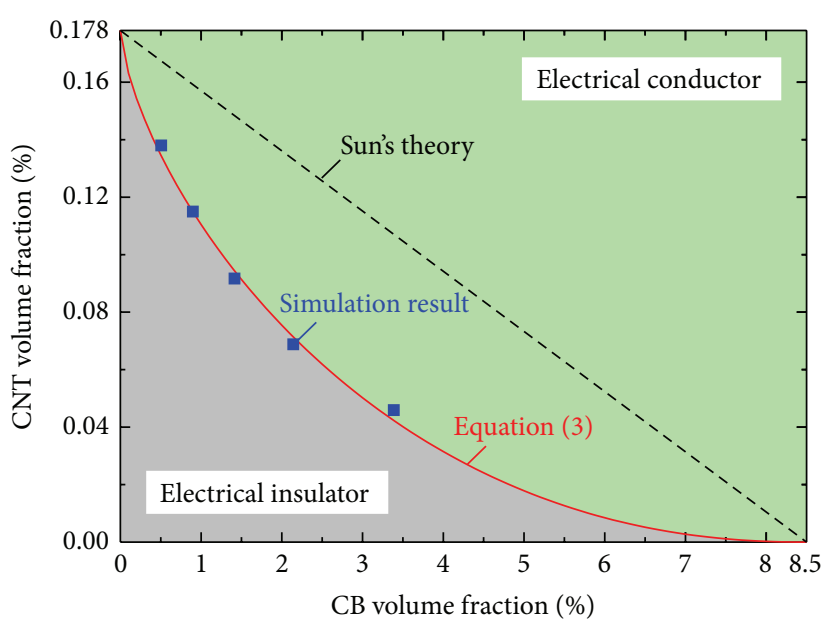

(b)

FIGURE 3: Synergistic effect of CB and CNT volume fractions: (a) effect of volume fractions on connection possibility and (b) relationship between volume fractions of $\mathrm{CB}$ and $\mathrm{CNT}$ at the percolation threshold.

to the theoretical results of percolation threshold, and the error between them becomes smaller with increasing CNT aspect ratio. When the aspect ratio goes beyond 1000, the error becomes less than $0.7 \%$. Even if the aspect ratio is as low as 300 , the error is still less than $8 \%$.

Comparing with experimental results, $\Phi_{0}$ is within the range of the scattered experimental data [9-14] and agrees with some experiments such as researches in [9]. In experiments, the percolation thresholds of CNTs with the same aspect ratio are usually higher than the simulation and theoretical values mainly because of the bad dispersion conditions.

From the comparison, it is revealed that the filler volume fraction for $50 \%$ connection possibility $\left(\Phi_{0}\right)$ can be considered as the electrical percolation threshold. And this choice is also physically valid, because when the connection possibility is greater than $50 \%$, the pathway has more chance to be formed and the composites are more inclined to be conductive, and when it is less than $50 \%$, the composites tend to be electrically isolated. So the volume fraction corresponding to $50 \%$ connection possibility is defined as the threshold at which the electrical conductivity of materials converts.

\section{Effects on Electrical Percolation Threshold}

When producing PMCs with CB-CNT hybrid filler, what concern us mostly is how much the hybrid filler is needed to transfer isolative polymer matrix into conductor; in other words, the percolation threshold is usually measured by the total volume fraction of CB and CNT hybrid fillers. Generally, the threshold depends on amounts and dimensions of $\mathrm{CB}$ and CNTs. So it is necessary to study the effects of volume fractions of $\mathrm{CB}$ and $\mathrm{CNT}$ on each other as well as the effects of dimensions of $\mathrm{CB}$ and $\mathrm{CNT}$ including the diameter of $\mathrm{CB}$ groups and the aspect ratio of CNTs.

It has been confirmed that the filler volume fraction for $50 \%$ connection possibility $\left(\Phi_{0}\right)$ obtained from fitting of Boltzmann function can be used to estimate the electrical percolation threshold. Then effects of $\mathrm{CB}$ and $\mathrm{CNT}$ on the percolation threshold for PMCs with CB-CNT hybrid fillers can be studied using the numerical method developed in Section 2. Firstly, a basic law for the synergistic effect of $\mathrm{CB}$ and $\mathrm{CNT}$ volume fractions on the percolation threshold is revealed, and then the effects of CNT aspect ratio and diameter ratio of $\mathrm{CB}$ groups to $\mathrm{CNTs}$ on the basic law are studied, respectively.

3.1. Synergistic Effect of CB and CNT Volume Fractions. In this study, the diameter and length of CNT models are $d_{\mathrm{CNT}}=$ $2 \mathrm{~nm}$ and $l_{\mathrm{CNT}}=600 \mathrm{~nm}$, respectively, and the diameter of $\mathrm{CB}$ models is $d_{\mathrm{CB}}=60 \mathrm{~nm}$. Thus, the percolation threshold is $\Phi_{0}^{\mathrm{CNT}}=0.178 \%$ for PMCs with CNT filler only, and $\Phi_{0}^{\mathrm{CB}}=$ $8.50 \%$ for PMCs with CB filler only. When the CNT volume fraction $\widehat{V}_{\mathrm{CNT}}=0.25 \Phi_{0}^{\mathrm{CNT}}, 0.375 \Phi_{0}^{\mathrm{CNT}}, 0.5 \Phi_{0}^{\mathrm{CNT}}, 0.625 \Phi_{0}^{\mathrm{CNT}}$, and $0.75 \Phi_{0}^{\mathrm{CNT}}$, the connection possibility of the PMCs with CB-CNT hybrid filler increases with the increasing $\mathrm{CB}$ volume fraction, following Boltzmann function, as shown in Figure 3(a). The $\mathrm{CB}$ volume fractions at the percolation threshold, denoted by $\widehat{V}_{\mathrm{CB}}$, are obtained when the connection possibility is $50 \%$. It is found that the volume fractions of $\mathrm{CB}$ and $\mathrm{CNT}$ at the percolation threshold follow the relation below as shown in Figure 3(b):

$$
\left(1-\frac{\widehat{V}_{\mathrm{CNT}}}{\Phi_{0}^{\mathrm{CNT}}}\right)^{a}+\left(1-\frac{\widehat{V}_{\mathrm{CB}}}{\Phi_{0}^{\mathrm{CB}}}\right)^{b}=1 .
$$


TABLE 3: Exponents $a$ and $b$ for different CNT aspect ratio $l_{\mathrm{CNT}} / d_{\mathrm{CNT}}$.

\begin{tabular}{lcc}
\hline$l_{\mathrm{CNT}} / d_{\mathrm{CNT}}$ & $a$ & $b$ \\
\hline 150 & 1.445 & 2.016 \\
200 & 1.506 & 2.160 \\
250 & 1.520 & 2.150 \\
300 & 1.478 & 2.179 \\
\hline
\end{tabular}

Here the exponents $a$ and $b$ are determined by dimensions of $\mathrm{CB}$ and CNT, and for the curve in Figure 3(b) they are 1.478 and 2.179 , respectively.

If no synergistic effect exists between $\mathrm{CB}$ and CNT volume fractions, based on Sun et al's study in 2009 [29], the relation equation should be linear as below:

$$
\frac{\widehat{V}_{\mathrm{CNT}}}{\Phi_{0}^{\mathrm{CNT}}}+\frac{\widehat{V}_{\mathrm{CB}}}{\Phi_{0}^{\mathrm{CB}}}=1 .
$$

It can be seen clearly from Figure 3(b) that the CB volume fraction needed to reach the composite threshold is much lower in our simulation than that in Sun's theory [29] (4). This nonlinear behavior and reduced volume fraction imply the existence of synergistic effect, consistent with the experimental observations $[1,30]$. It should be pointed out that when $\widehat{V}_{\mathrm{CNT}} / \Phi_{0}^{\mathrm{CNT}} \ll 1$ or $\widehat{V}_{\mathrm{CB}} / \Phi_{0}^{\mathrm{CB}} \ll 1$ the difference between Sun's theory [29] and (3) is tiny and negligible, so in [29] and (4) is valid because $\widehat{V}_{\mathrm{CB}} / \Phi_{0}^{\mathrm{CB}} \ll 1$.

Figure 3(b) also shows that when the CNT volume fraction $\widehat{V}_{\mathrm{CNT}}$ is close to $\Phi_{0}^{\mathrm{CNT}}$, increasing CB volume fraction $\left(\widehat{V}_{\mathrm{CB}}\right)$ can greatly decrease $\widehat{V}_{\mathrm{CNT}}$, which means the $\mathrm{CB}$ volume fraction has significant effect on the CNT volume fraction at the percolation threshold. However, if the CNT volume fraction $\left(\widehat{V}_{\mathrm{CNT}}\right)$ is much lower than $\Phi_{0}^{\mathrm{CNT}}$, adding $\mathrm{CB}$ can hardly transform electrically isolating polymers to conductors, until CB volume fraction $\widehat{V}_{\mathrm{CB}}$ is very close to $\Phi_{0}^{\mathrm{CB}}$. This conclusion agrees with the experiment results of Socher et al. [1].

3.2. Effect of CNT Aspect Ratio. In (3) of the relation between $\mathrm{CB}$ and $\mathrm{CNT}$ volume fraction at filler percolation threshold, the parameters $a$ and $b$ are determined by dimensions of CNTs and CB groups. It has been proved that for CNTs the aspect ratio is the dominated factor that affects the percolation threshold in PMCs with only CNT filler [15]; in other words, it affects $\Phi_{0}^{\mathrm{CNT}}$ significantly. However, in the PMCs with $\mathrm{CB}$ and CNT hybrid fillers, whether the CNT aspect ratio affects the relation between the volume fractions of $\mathrm{CB}$ and $\mathrm{CNT}$ at the percolation threshold established in (3) and how it affects the exponents $a$ and $b$ of (3) are still unrevealed. So in this subsection, the effect of CNT aspect ratio on (3) as well as the exponents $a$ and $b$ is studied.

In the simulation, the diameters of $\mathrm{CB}$ groups and CNTs are set to be $d_{\mathrm{CB}}=60 \mathrm{~nm}$ and $d_{\mathrm{CNT}}=2 \mathrm{~nm}$, respectively. In order to get different $\mathrm{CNT}$ aspect ratios, the lengths of CNT in the simulation are $l_{\mathrm{CNT}}=300 \mathrm{~nm}, 400 \mathrm{~nm}, 500 \mathrm{~nm}$, and $600 \mathrm{~nm}$, corresponding to the aspect ratio of 150, 200,
250, and 300. And for these different aspect ratios $\Phi_{0}^{\mathrm{CNT}}$ can be obtained as $0.390 \%, 0.284 \%, 0.224 \%$, and $0.178 \%$. CNT volume fractions $\widehat{V}_{\mathrm{CNT}}=0.25 \Phi_{0}^{\mathrm{CNT}}, 0.375 \Phi_{0}^{\mathrm{CNT}}, 0.5 \Phi_{0}^{\mathrm{CNT}}$, $0.625 \Phi_{0}^{\mathrm{CNT}}$, and $0.75 \Phi_{0}^{\mathrm{CNT}}$ are studied, respectively, for every different aspect ratio.

The simulation results of $\mathrm{CB}$ and CNT volume fractions at percolation threshold are shown in Figure 4(a). In Figure 4(a), for each CNT aspect ratio, the data can be fitted by (3) very well, as shown by the solid curves. The parameters $a$ and $b$ for each aspect ratio are listed in Table 3. It is found that the aspect ratio of CNT can barely affect $a$ or $b$. This conclusion can be better illustrated in Figure 4(b), in which the CNT and CB volume fractions are normalized by $\Phi_{0}^{\mathrm{CNT}}$ and $\Phi_{0}^{\mathrm{CB}}$, respectively. In Figure 4(b), all the fitted curves are very close to each other especially for those with CNT aspect ratio greater than 200. In practical applications, the aspect ratio of CNTs is usually greater than 1000 . So the CB-CNT hybrid filled PMCs with different CNT aspect ratios follow the same threshold rule and the exponents $a$ and $b$ can be considered as unchanged for different CNT aspect ratios. It should be noted that increasing CNT aspect ratio can decrease $\Phi_{0}^{\mathrm{CNT}}$ and hence decrease the percolation threshold of composites with hybrid filler, although the CNT aspect ratio has little effect on the parameters $a$ and $b$.

3.3. Effect of Diameter Ratio. In PMCs with CB-CNT hybrid filler, $\mathrm{CB}$ can be very different in size due to the differences in processing, dispersion method, dispersion time, and so forth. The diameter ratio of CB groups to CNT in hybrid filler may affect the composite percolation threshold. Here the diameter and length of CNTs are set to be $d_{\mathrm{CNT}}=2 \mathrm{~nm}$ and $l_{\mathrm{CNT}}=600 \mathrm{~nm}$, respectively, and the CB groups with different diameters $\left(d_{\mathrm{CB}}\right)$ of $40 \mathrm{~nm}, 50 \mathrm{~nm}$, $60 \mathrm{~nm}, 70 \mathrm{~nm}$, and $80 \mathrm{~nm}$ corresponding to the diameter ratios $\left(d_{\mathrm{CB}} / d_{\mathrm{CNT}}\right)$ of $20,25,30,35$, and 40 are studied. For each diameter ratio, the volume fractions of CNT and $\mathrm{CB}$ at the composite percolation threshold are obtained by studying the connection possibility and presented in Figure 5(a).

From Figure 5(a) it can be observed that for a given CNT volume fraction the $\mathrm{CB}$ volume fraction at percolation threshold $\widehat{V}_{\mathrm{CB}}$ is reduced by increasing the diameter ratio $d_{\mathrm{CB}} / d_{\mathrm{CNT}}$. And this reduction effect becomes more significant with the decrease of the pregiven CNT volume fraction. So increasing the diameter of $\mathrm{CB}$ groups can decrease the composite percolation threshold, especially when the CNT volume fraction is relatively low, but it is not a good way to get low percolation threshold by producing large CB groups for large agglomeration which may lead to decline in other properties of the composites.

It can also be illuminated in Figure 5(a) that relation between the volume fractions of $\mathrm{CB}$ and $\mathrm{CNT}$ follows the rule of (3), as shown by the fitted solid curves in Figure 5(a). The parameters $a$ and $b$ of these five fitting curves are exhibited in Figure 5(b). It is found that the exponent $a$ is almost not affected by the diameter ratio while the exponent $b$ increases with increasing diameter ratio. Furthermore, 


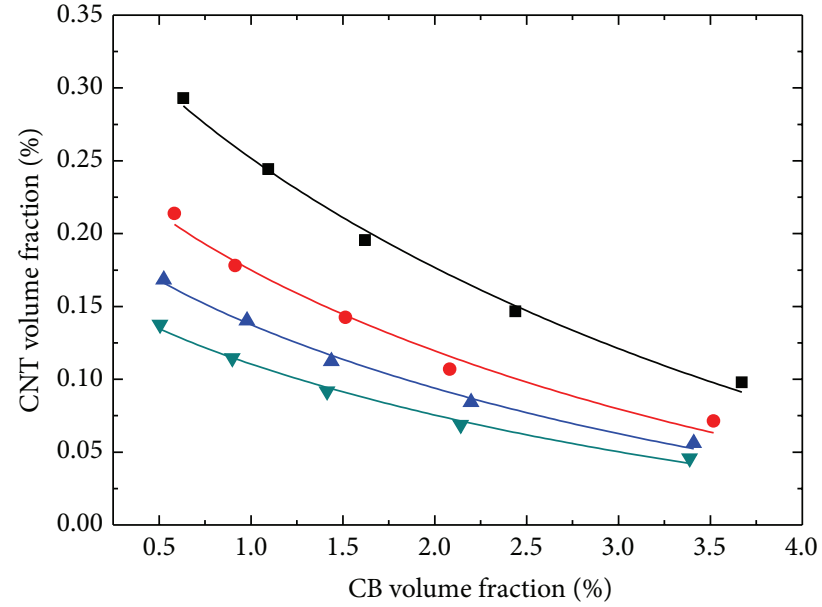

- $l_{\mathrm{CNT}} / d_{\mathrm{CNT}}=150$

- $l_{\mathrm{CNT}} / d_{\mathrm{CNT}}=200$
- $l_{\mathrm{CNT}} / d_{\mathrm{CNT}}=250$

$\nabla l_{\mathrm{CNT}} / d_{\mathrm{CNT}}=300$

(a)

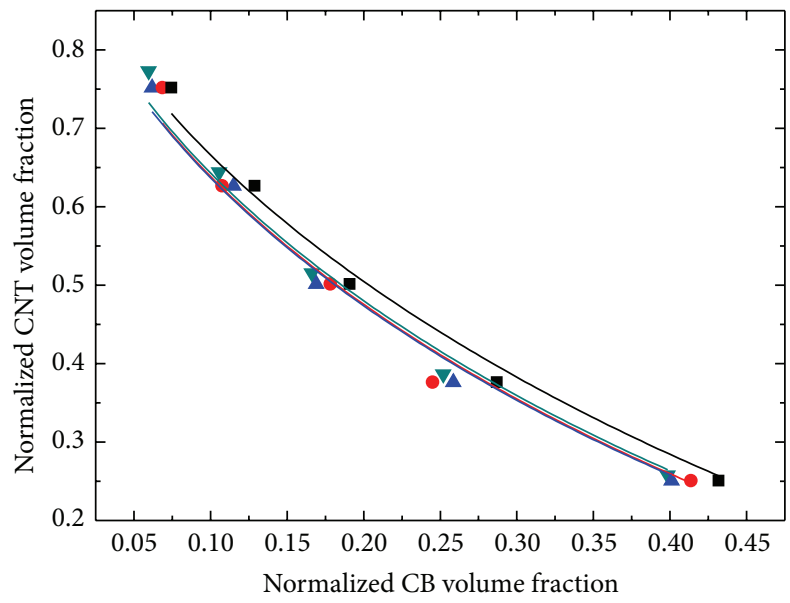

- $l_{\mathrm{CNT}} / d_{\mathrm{CNT}}=150$

- $l_{\mathrm{CNT}} / d_{\mathrm{CNT}}=200$
$\Delta l_{\mathrm{CNT}} / d_{\mathrm{CNT}}=250$ $\checkmark l_{\mathrm{CNT}} / d_{\mathrm{CNT}}=300$

(b)

FIGURE 4: Relation between CB and CNT volume fraction at filler percolation threshold for different CNT aspect ratios: (a) volume fractions of $\mathrm{CB}$ and $\mathrm{CNT}$ and (b) $\mathrm{CB}$ and $\mathrm{CNT}$ volume fractions normalized by $\Phi_{0}^{\mathrm{CNT}}$ and $\Phi_{0}^{\mathrm{CB}}$, respectively.
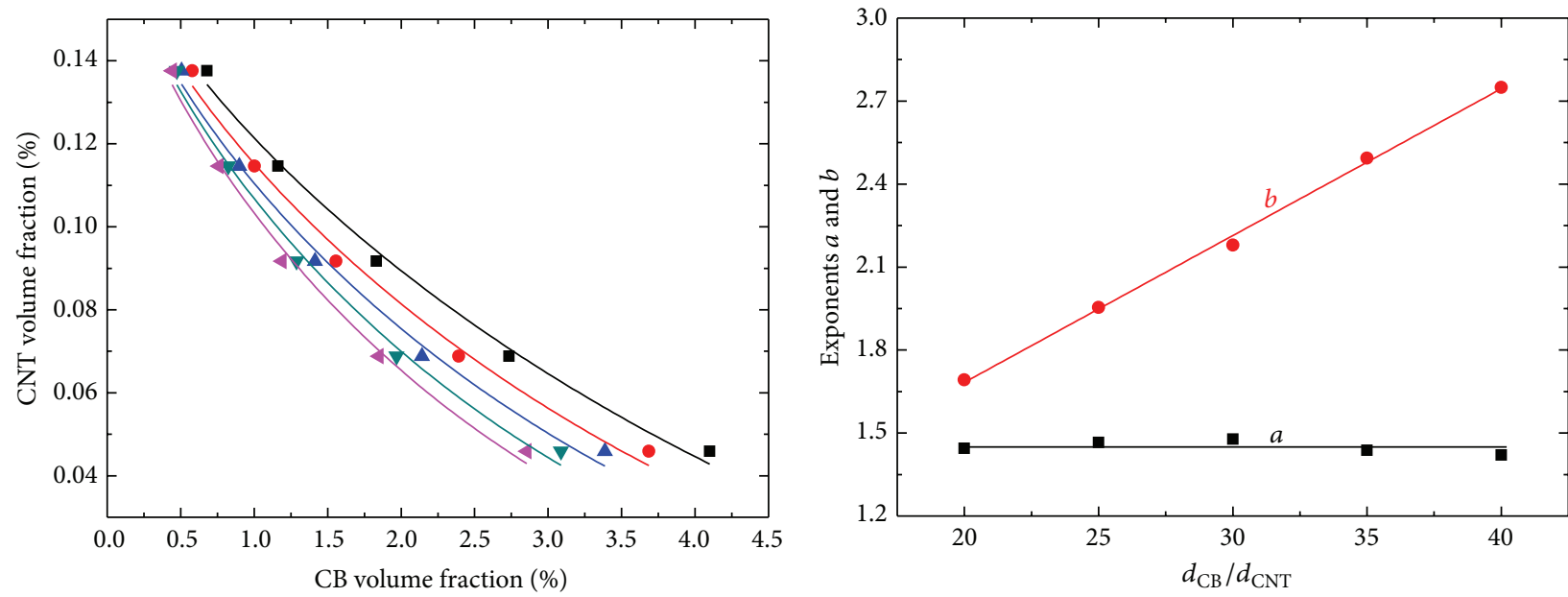

- $d_{\mathrm{CB}} / d_{\mathrm{CNT}}=20$

- $d_{\mathrm{CB}} / d_{\mathrm{CNT}}=25$

- $d_{\mathrm{CB}} / d_{\mathrm{CNT}}=30$

$\nabla d_{\mathrm{CB}} / d_{\mathrm{CNT}}=35$

$<d_{\mathrm{CB}} / d_{\mathrm{CNT}}=40$

(a)

(b)

FIGURE 5: Effect of diameter ratio: (a) relation between CB and CNT volume fractions at filler percolation threshold for different diameter ratios of CB to CNT and (b) relation between exponents $a$ and $b$ and diameter ratio of CB to CNT.

it is reasonable to consider that the relation between exponent $b$ and the diameter ratio $d_{\mathrm{CB}} / d_{\mathrm{CNT}}$ is linear following the equation below:
Thus (3) can be rewritten by replacing $a$ by its average value and $b$ by (5) as below:

$$
\left(1-\frac{\widehat{V}_{\mathrm{CNT}}}{\Phi_{0}^{\mathrm{CNT}}}\right)^{1.449}+\left(1-\frac{\widehat{V}_{\mathrm{CB}}}{\Phi_{0}^{\mathrm{CB}}}\right)^{0.622+0.0531\left(d_{\mathrm{CB}} / d_{\mathrm{CNT}}\right)}=1 .
$$




\section{Estimation on Electrical Percolation Threshold}

It has been revealed above that in PMCs with hybrid fillers the relation between volume fractions of $\mathrm{CB}$ and $\mathrm{CNT}$ at percolation threshold depends on their own percolation threshold in PMCs with single filler and their volume fractions as well as their diameter ratio. The CNT aspect ratio can only affect the percolation threshold in PMCs with only CNTs $\Phi_{0}^{\mathrm{CNT}}$. Therefore, the composite percolation threshold $\Phi_{0}^{\mathrm{CB} \& \mathrm{CNT}}$, which is defined as the volume fraction of both fillers when the electrical conductivity of the composites converts, can be estimated by replacing $\widehat{V}_{\mathrm{CNT}}$ by $\left(\Phi_{0}^{\mathrm{CB} \& \mathrm{CNT}}-\widehat{V}_{\mathrm{CB}}\right)$ as

$$
\begin{aligned}
& \Phi_{0}^{\mathrm{CB} \& \mathrm{CNT}} \\
& =\Phi_{0}^{\mathrm{CNT}}\left\{1-\left[1-\left(1-\frac{\widehat{V}_{\mathrm{CB}}}{\Phi_{0}^{\mathrm{CB}}}\right)^{0.622+0.0531\left(d_{\mathrm{CB}} / d_{\mathrm{CNT}}\right)}\right]^{0.690}\right\} \\
& \quad+\widehat{V}_{\mathrm{CB}} .
\end{aligned}
$$

Similarly, $\Phi_{0}^{\mathrm{CB} \& C N T}$ can also be estimated by the CNT volume fraction $\widehat{V}_{\mathrm{CNT}}$ as

$$
\begin{aligned}
& \Phi_{0}^{\mathrm{CB} \& \mathrm{CNT}} \\
& =\Phi_{0}^{\mathrm{CB}}\left\{1-\left[1-\left(1-\frac{\widehat{V}_{\mathrm{CNT}}}{\Phi_{0}^{\mathrm{CNT}}}\right)^{1.449}\right]^{d_{\mathrm{CNT}} /\left(0.622 d_{\mathrm{CNT}}+0.0531 d_{\mathrm{CB}}\right)}\right\} \\
& +\widehat{V}_{\mathrm{CNT}}
\end{aligned}
$$

In (7) and (8), the percolation thresholds of PMCs with single filler, $\Phi_{0}^{\mathrm{CNT}}$ and $\Phi_{0}^{\mathrm{CB}}$, can be obtained by many existing methods, such as excluded volume method and Monte Carlo simulation, and the diameters and volume fractions of $\mathrm{CB}$ and CNTs are determined by material processing.

\section{Conclusions}

The electrical percolation threshold of PMCs with hybrid filler system of CNT and CB is studied by Monte Carlo simulation on the connection possibility of the fillers. In the simulation, CNTs are modeled as slender spherocylinders and agglomerated CB particles are modeled as hypothetical spheres with interspaces. The overlapped volume is deducted to deal with the impenetrability among CNTs. The following conclusions can be drawn in this study.

(1) The electrical percolation threshold can be defined as the filler volume fraction at $50 \%$ connection possibility, which is not only physical valid but also verified by theoretical and experimental results.

(2) The synergistic effect exists in PMCs with CB-CNT hybrid filler system, and the $\mathrm{CB}$ and $\mathrm{CNT}$ volume fractions at composite percolation threshold follow the nonlinear relation in (6).

(3) Adding CB into CNT composites can decrease the CNT volume needed to convert the composite conductivity only when the CNT volume fraction is close to $\Phi_{0}^{\mathrm{CNT}}$, the threshold of PMCs with only CNT. If the CNT volume fraction is very low, adding CB is not a good way to make the conversion.

(4) The electrical percolation threshold can be decreased by increasing CNT aspect ratio, as well as increasing the diameter ratio of CB groups to CNTs, but the latter is not an effective way because large agglomeration of $\mathrm{CB}$ particles may lead to decline in other properties of the composites.

(5) In practice applications, the percolation threshold of PMCs with CB-CNT hybrid filler can be estimated formularily by (7) (or (8)) if CB (or CNT) volume fraction is required.

\section{Conflict of Interests}

The authors declare that there is no conflict of interests regarding the publishing of this paper.

\section{Acknowledgments}

The work was supported by the National Natural Science Foundation of China (no. 11202012) and the Program for New Century Excellent Talents in University (no. NCET-13-0021).

\section{References}

[1] R. Socher, B. Krause, S. Hermasch, R. Wursche, and P. Pötschke, "Electrical and thermal properties of polyamide 12 composites with hybrid fillers systems of multiwalled carbon nanotubes and carbon black," Composites Science and Technology, vol. 71, no. 8, pp. 1053-1059, 2011.

[2] J. N. Coleman, S. Curran, A. B. Dalton et al., "Percolationdominated conductivity in a conjugated-polymer-carbonnanotube composite," Physical Review B-Condensed Matter and Materials Physics, vol. 58, no. 12, pp. R7492-R7495, 1998.

[3] Y. L. Chen, B. Liu, X. Q. He, Y. Huang, and K. C. Hwang, "Failure analysis and the optimal toughness design of carbon nanotubereinforced composites," Composites Science and Technology, vol. 70, no. 9, pp. 1360-1367, 2010.

[4] J. Sandler, M. S. P. Shaffer, T. Prasse, W. Bauhofer, K. Schulte, and A. H. Windle, "Development of a dispersion process for carbon nanotubes in an epoxy matrix and the resulting electrical properties," Polymer, vol. 40, no. 21, pp. 5967-5971, 1999.

[5] J. K. W. Sandler, J. E. Kirk, I. A. Kinloch, M. S. P. Shaffer, and A. H. Windle, "Ultra-low electrical percolation threshold in carbon-nanotube-epoxy composites," Polymer, vol. 44, no. 19, pp. 5893-5899, 2003.

[6] B. Krause, M. Ritschel, C. Täschner et al., "Comparison of nanotubes produced by fixed bed and aerosol-CVD methods and their electrical percolation behaviour in melt mixed polyamide 6.6 composites," Composites Science and Technology, vol. 70, no. 1, pp. 151-160, 2010. 
[7] W. Bauhofer and J. Z. Kovacs, "A review and analysis of electrical percolation in carbon nanotube polymer composites," Composites Science and Technology, vol. 69, no. 10, pp. 14861498, 2009.

[8] P. C. Ma, M. Y. Liu, H. Zhang et al., "Enhanced electrical conductivity of nanocomposites containing hybrid fillers of carbon nanotubes and carbon black," ACS Applied Materials and Interfaces, vol. 1, no. 5, pp. 1090-1096, 2009.

[9] J. Z. Kovacs, B. S. Velagala, K. Schulte, and W. Bauhofer, "Two percolation thresholds in carbon nanotube epoxy composites," Composites Science and Technology, vol. 67, no. 5, pp. 922-928, 2007.

[10] H. Koerner, W. D. Liu, M. Alexander, P. Mirau, H. Dowty, and R. A. Vaia, "Deformation-morphology correlations in electrically conductive carbon nanotube-thermoplastic polyurethane nanocomposites," Polymer, vol. 46, no. 12, pp. 4405-4420, 2005.

[11] A. Moisala, Q. Li, I. A. Kinloch, and A. H. Windle, "Thermal and electrical conductivity of single- and multi-walled carbon nanotube-epoxy composites," Composites Science and Technology, vol. 66, no. 10, pp. 1285-1288, 2006.

[12] F. H. Gojny, M. H. G. Wichmann, B. Fiedler et al., "Evaluation and identification of electrical and thermal conduction mechanisms in carbon nanotube/epoxy composites," Polymer, vol. 47, no. 6, pp. 2036-2045, 2006.

[13] A. Allaoui, S. Bai, H. M. Cheng, and J. B. Bai, "Mechanical and electrical properties of a MWNT/epoxy composite," Composites Science and Technology, vol. 62, no. 15, pp. 1993-1998, 2002.

[14] L. Wang and Z. M. Dang, "Carbon nanotube composites with high dielectric constant at low percolation threshold," Applied Physics Letters, vol. 87, no. 4, Article ID 042903, 2005.

[15] I. Balberg, C. H. Anderson, S. Alexander, and N. Wagner, "Excluded volume and its relation to the onset of percolation," Physical Review B, vol. 30, no. 7, pp. 3933-3943, 1984.

[16] M. Grujicic, G. Cao, and W. N. Roy, "A computational analysis of the percolation threshold and the electrical conductivity of carbon nanotubes filled polymeric materials," Journal of Materials Science, vol. 39, no. 14, pp. 4441-4449, 2004.

[17] F. Du, J. E. Fischer, and K. I. Winey, "Effect of nanotube alignment on percolation conductivity in carbon nanotube/polymer composites," Physical Review B-Condensed Matter and Materials Physics, vol. 72, no. 12, Article ID 121404, 4 pages, 2005.

[18] L. Berhan and A. M. Sastry, "Modeling percolation in highaspect-ratio fiber systems. I. Soft-core versus hard-core models," Physical Review E-Statistical, Nonlinear, and Soft Matter Physics, vol. 75, no. 4, Article ID 041120, 8 pages, 2007.

[19] L. Berhan and A. M. Sastry, "Modeling percolation in highaspect-ratio fiber systems. II. the effect of waviness on the percolation onset," Physical Review E-Statistical, Nonlinear, and Soft Matter Physics, vol. 75, no. 4, Article ID 041121, 7 pages, 2007.

[20] A. E. Eken, E. J. Tozzi, D. J. Klingenberg, and W. Bauhofer, "A simulation study on the combined effects of nanotube shape and shear flow on the electrical percolation thresholds of carbon nanotube/polymer composites," Journal of Applied Physics, vol. 109, no. 8, Article ID 084342, 2011.

[21] W. S. Bao, S. A. Meguid, Z. H. Zhu, Y. Pan, and G. J. Weng, "A novel approach to predict the electrical conductivity of multifunctional nanocomposites," Mechanics of Materials, vol. 46, pp. 129-138, 2012.

[22] N. Hu, Z. Masuda, C. Yan, G. Yamamoto, H. Fukunaga, and T. Hashida, "The electrical properties of polymer nanocomposites with carbon nanotube fillers," Nanotechnology, vol. 19, no. 21, Article ID 215701, 2008.

[23] C. Y. Li and T. W. Chou, "Continuum percolation of nanocomposites with fillers of arbitrary shapes," Applied Physics Letters, vol. 90, no. 17, Article ID 174108, 2007.

[24] W. B. Lu, T. W. Chou, and E. T. Thostenson, "A threedimensional model of electrical percolation thresholds in carbon nanotube-based composites," Applied Physics Letters, vol. 96, no. 22, Article ID 223106, 2010.

[25] W. Zhou, Y. Huang, B. Liu et al., "Self-folding of single- and multiwall carbon nanotubes," Applied Physics Letters, vol. 90, no. 7, Article ID 073107, 2007.

[26] W. B. Lu and T. W. Chou, "Analysis of the entanglements in carbon nanotube fibers using a self-folded nanotube model," Journal of the Mechanics and Physics of Solids, vol. 59, no. 3, pp. 511-524, 2011.

[27] M. B. Bryning, M. F. Islam, J. M. Kikkawa, and A. G. Yodh, "Very low conductivity threshold in bulk isotropic single-walled carbon nanotube-epoxy composites," Advanced Materials, vol. 17, no. 9, pp. 1186-1191, 2005.

[28] J. Sumfleth, X. C. Adroher, and K. Schulte, "Synergistic effects in network formation and electrical properties of hybrid epoxy nanocomposites containing multi-wall carbon nanotubes and carbon black," Journal of Materials Science, vol. 44, no. 12, pp. $3241-3247,2009$

[29] Y. Sun, H.-D. Bao, Z.-X. Guo, and J. Yu, "Modeling of the electrical percolation of mixed carbon fillers in polymer-based composites," Macromolecules, vol. 42, no. 1, pp. 459-463, 2009.

[30] M. Wen, X. Sun, L. Su, J. Shen, J. Li, and S. Guo, “The electrical conductivity of carbon nanotube/carbon black/polypropylene composites prepared through multistage stretching extrusion," Polymer, vol. 53, no. 7, pp. 1602-1610, 2012. 

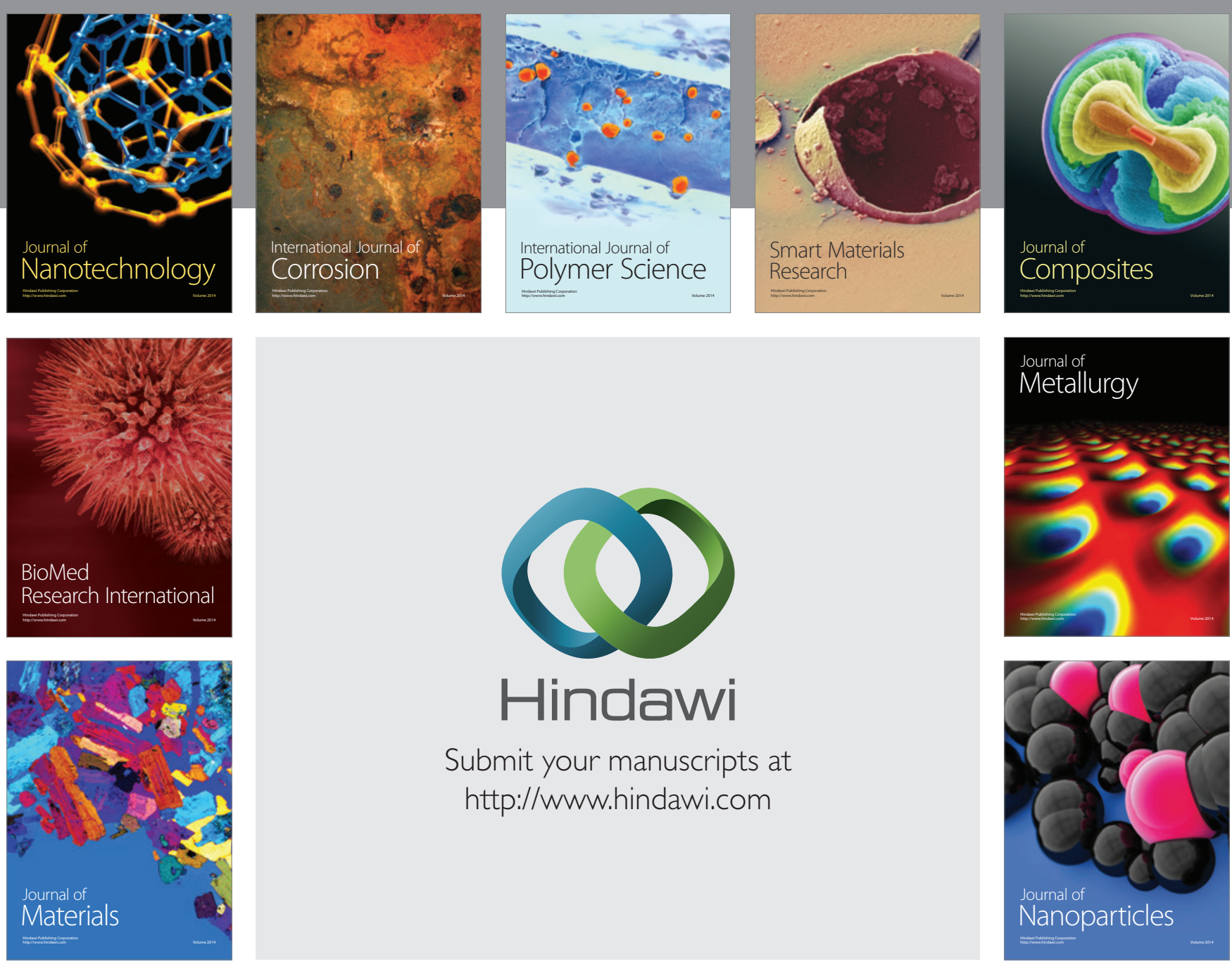

Submit your manuscripts at http://www.hindawi.com
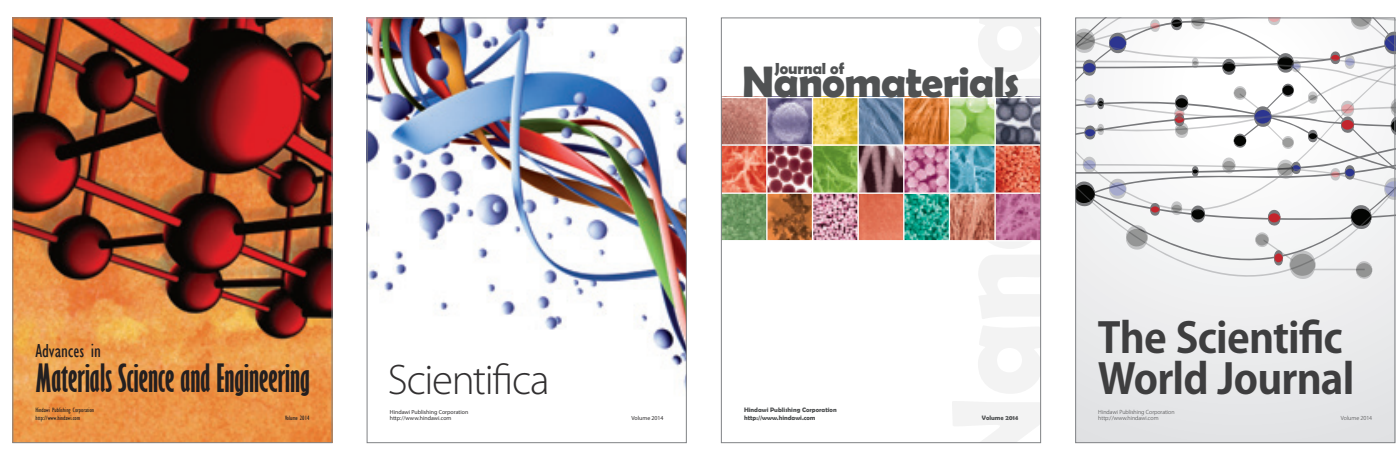

\section{The Scientific World Journal}
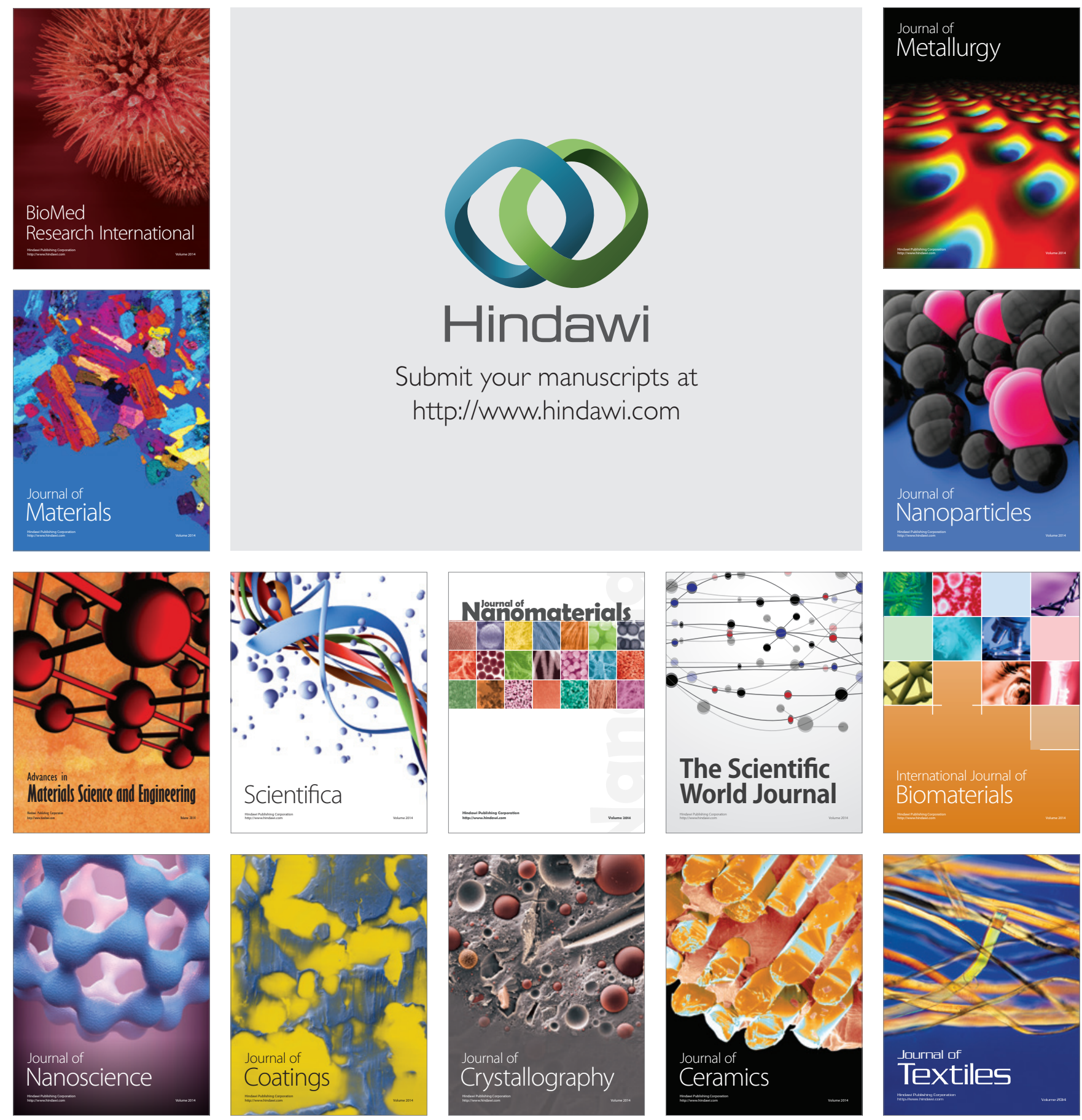